\title{
Probing the doubly charged Higgs boson with a muonium to antimuonium conversion experiment
}

\author{
Chengcheng Han, ${ }^{1}$ Da Huang $\odot,{ }^{2,3,4, *}$ Jian Tang ${ }^{1,},{ }^{\dagger}$ and Yu Zhang $\oplus^{5,6}$ \\ ${ }^{1}$ School of Physics, Sun Yat-Sen University, Guangzhou 510275, China \\ ${ }^{2}$ National Astronomical Observatories, Chinese Academy of Sciences, Beijing 100012, China \\ ${ }^{3}$ School of Fundamental Physics and Mathematical Sciences, Hangzhou Institute for Advanced Study, \\ University of Chinese Academy of Sciences, Hangzhou 310024, China \\ ${ }^{4}$ International Center for Theoretical Physics Asia-Pacific, Beijing/Hangzhou 10010, China \\ ${ }^{5}$ Institutes of Physical Science and Information Technology, Anhui University, Hefei 230601, China \\ ${ }^{6}$ School of Physics and Materials Science, Anhui University, Hefei 230601, China
}

(Received 8 February 2021; accepted 10 March 2021; published 30 March 2021)

\begin{abstract}
The spontaneous muonium-to-antimuonium conversion is one of the interesting charged lepton flavor violation processes. The Muonium-to-Antimuonium Conversion Experiment (MACE) is the next generation experiment to probe such a phenomenon. In models with a triplet Higgs boson to generate neutrino masses, such as the type-II seesaw and its variant, this process can be induced by the doubly charged Higgs boson contained in it. In this article, we study the prospect of MACE to probe these models via the muonium-to-antimuonium transitions. After considering the limits from $\mu^{+} \rightarrow e^{+} \gamma$ and $\mu^{+} \rightarrow e^{+} e^{-} e^{+}$, we find that MACE could probe a parameter space for the doubly charged Higgs boson, which is beyond the reach of LHC and other flavor experiments.
\end{abstract}

DOI: 10.1103/PhysRevD.103.055023

\section{INTRODUCTION}

The observation of neutrino oscillations has indicated that neutrinos have very tiny but nonzero masses, which is one of the direct evidences towards the new physics (NP) beyond the Standard Model (SM). Traditionally, the explanation of the measured neutrino masses and mixings involves the seesaw mechanism, where the smallness of neutrino masses is allowed with the existence of heavy eigenstates. One of the most striking predictions of the seesaw models is the existence of charged lepton flavor violation (cLFV) processes. Indeed, the neutrino oscillations can be viewed as a manifestation of the neutral lepton flavor violation (LFV). Therefore, it is natural to think about the corresponding LFV in the charged lepton channels. Currently, there are many ongoing and upcoming experiments searching for the cLFV processes all over the world. The accelerator muon beam experiments at PSI are searching for $\mu^{+} \rightarrow e^{+} e^{+} e^{-}$by the Mu3e Collaboration [1]

\footnotetext{
*Corresponding author. dahuang@bao.ac.cn

Corresponding author. tangjian5@mail.sysu.edu.cn

Published by the American Physical Society under the terms of the Creative Commons Attribution 4.0 International license. Further distribution of this work must maintain attribution to the author(s) and the published article's title, journal citation, and DOI. Funded by SCOAP ${ }^{3}$.
}

and for $\mu^{+} \rightarrow e^{+} \gamma$ by MEG-II [2]. Furthermore, the coherent muon-to-electron conversions $\left(\mu^{-} N \rightarrow e^{-} N\right)$ will be searched for by COMET [3] at J-PARC and Mu2e [4] at FNAL, both of which are still under construction.

Another equally important but less studied cLFV channel is the muonium-to-antimuonium conversion, which was first brought forward by Pontecorvo [5] more than half a century ago. Muonium $M$ is a hydrogenlike bound state $\left(e^{-} \mu^{+}\right)$formed by an electron and an antimuon, while an antimuonium $\bar{M}$ is the corresponding antiparticle bounded by a positron and a muon $\left(e^{+} \mu^{-}\right)$. Experimentally, one can produce muonium atoms by colliding slow muons into a target material and then trying to see the spontaneous conversion from muoniums into antimuoniums. In the SM, such a conversion is forbidden by the lepton flavor symmetry. Hence, the detection of this process can be viewed as a probe to the physics beyond the SM. In light of its potential significance, there have been many theoretical studies on the muonium-to-antimuonium transition in some well-motivated models, such as the type-I [6-8] and type-II seesaw [9] models, the $Z_{8}$ model with more than four lepton generations [10], the minimal 331 model [11], the R-parity violated SUSY model [12], and the left-right symmetric model [13], as well as the low-energy effective operator framework [14]. The complementary study between low-energy and high-energy phenomenonology in LFVs was well explored in terms of a doubly charged scalar in Refs. $[15,16]$, while more attention was paid to the type-II seesaw scalar triplet model at a high-energy 
proton-proton collider $[17,18]$. More recently, the $M-\bar{M}$ conversion was explored theoretically in a modelindependent way in Ref. [19].

At present, the best upper constraint on the muonium-toantimuonium conversion has been given by the PSI experiment in 1999, which presented the upper limit on the conversion probability to be $\mathcal{P}(M \leftrightarrow \bar{M}) \lesssim 8.3 \times 10^{-11}$ at the $90 \%$ confidence level [20]. During the past two decades, there has not been any experimental improvement in this important cLFV channel. However, this situation is expected to soon change in the near future due to the advent of the Muonium-to-Antimuonium Conversion Experiment (MACE) in China [21]. MACE is the next-generation experiment specialized to measure this exceptional cLFV process, which will be operated at the upgraded $500 \mathrm{~kW}$ pulsed proton accelerator running at the China Spallation Neutron Source (CSNS). By taking advantage of the highquality intense slow muon sources with more than $10^{8} \mu^{+}$ produced per second and the beam spread smaller than 5\% at CSNS, together with the high-efficiency muonium formation targets, the high-precision magnetic spectrometer, and the optimized detector setup by locating the detector at the front end of a future muon collider, MACE is expected to enhance the sensitivity to the $M-\bar{M}$ conversion probability by more than 2 orders of magnitude from the existing PSI experiment. Therefore, we expect that MACE can play an important role in probing and constraining the parameter space of NP models to generate the neutrino masses.

In light of promising experimental developments projected by MACE, it is timely to investigate the muoniumto-antimuonium conversion in more detail, paying attention to its interplay with other flavor and collider searches in probing the model parameter space of interest. In the present paper, we shall explore this aspect in the type-II seesaw model and its variant as a case study. The traditional type-II seesaw model generates the small active neutrino masses by introducing a $S U(2)_{L}$ triplet scalar, in which the doubly charged scalar component can induce the muoniumto-antimuonium conversion at tree level. Note that, due to the model structure in the type-II seesaw, the predicted $M-\bar{M}$ conversion probability is intimately correlated to the neutrino oscillation data and other cLFV processes, such as $\mu^{+} \rightarrow e^{-} e^{+} e^{+}$and $\mu^{+} \rightarrow e^{+} \gamma$. As a result, it will be seen below that MACE is not sensitive to the relevant parameter space surviving after imposing the existing cFLV constraints. In other words, if MACE can observe the positive signal on the muonium-to-antimuonium transition, it means that the simplest type-II seesaw model is ruled out, and one is required to consider some extensions to it. In our discussion, we shall provide such an example by incorporating in the type-II seesaw model only a single heavy right-handed neutrino, which will be called the hybrid seesaw model in the following. It turns out that MACE can provide additional information in this hybrid model on the parameter regions, which cannot be reached by other experiments.

This article is organized as follows: in Sec. II, we briefly describe quantum mechanics of the muonium-to-antimuonium transition based on the effective field theory and then estimate the MACE sensitivity to this process. In Sec. III, we give a brief introduction to the type-II and hybrid seesaw models. Then we detail the leading-order calculation of the $M-\bar{M}$ conversion probability in these two models. Section IV is devoted to the discussion of existing constraints on the type-II and hybrid seesaw models, including those from the measured neutrino masses and mixings, other cLFV processes, and collider searches on the doubly charged scalar. We present our numerical results in Sec. V. Finally, we summarize and conclude in Sec. VI.

\section{MACE PROSPECTS ON THE MUONIUM- ANTIMUONIUM TRANSITIONS}

Muonium $|M\rangle$ is a nonrelativistic Coulombic bound state of $\mu^{+}$and $e^{-}$, and antimuonium $|\bar{M}\rangle$ is a similar bound state of $\mu^{-}$and $e^{+}$. The nontrivial mixing between $|M\rangle$ and $|\bar{M}\rangle$ implies the nonvanishing lepton flavor violation (LFV) amplitude for $e^{-} \mu^{+} \rightarrow e^{+} \mu^{-}$.

Currently, the most precise measurement of the conversion probability of the $M-\bar{M}$ conversion was given by the PSI Collaboration two decades ago, with $P(M \leftrightarrow \bar{M})<$ $8.3 \times 10^{-11}$ at $95 \%$ C.L. The MACE Collaboration at CSNS attempts to improve the sensitivity of this conversion to the level of $P(M \leftrightarrow \bar{M}) \sim \mathcal{O}\left(10^{-14}\right)$.

Microscopically, the transition between $M$ and $\bar{M}$ can be described by the following local effective Hamiltonion density [7]:

$\mathcal{H}_{\mathrm{eff}}=\frac{G_{M \bar{M}}}{\sqrt{2}}\left[\bar{\mu}(x) \gamma^{\alpha}\left(1-\gamma^{5}\right) e(x)\right]\left[\bar{\mu}(x) \gamma_{\alpha}\left(1-\gamma^{5}\right) e(x)\right]$,

where $G_{M \bar{M}}$ is the corresponding Wilson coefficient. Due to the LFV transition, $|M\rangle$ and $|\bar{M}\rangle$ are not mass eigenstates. In this basis, the mass matrix has nondiagonal components,

$$
m_{M \bar{M}}=\left\langle M\left|\int d^{3} x \mathcal{H}_{\mathrm{eff}}(\vec{x})\right| \bar{M}\right\rangle,
$$

which can be transformed into the form of its constituents with a momentum distribution $f(p)$,

$$
|M(0)\rangle=\int \frac{d^{3} p}{(2 \pi)^{3}} f(p) a_{e}^{\dagger}(p, s) b_{\mu}^{\dagger}(-p, s)|0\rangle,
$$

where $a_{e}^{\dagger}(p, s)$ creates a fermion $e^{-}$with energy $+E_{p}$ and momentum $\vec{p}$ and $b_{\mu}^{\dagger}(-p, s)$ creates an antiparticle $\mu^{+}$with the opposite momentum and the same energy $+E_{p}$. If we neglect the momentum dependence in the spinors, the mass mixing element is 


$$
m_{M \bar{M}}=16 \times \frac{G_{M \bar{M}}}{\sqrt{2}}\left|\int \frac{d^{3} p}{(2 \pi)^{3}} f(p)\right|^{2}\left[\bar{u}_{L}^{(\mu)} \gamma^{\alpha} u_{L}^{(e)} \bar{v}_{L}^{(\mu)} \gamma_{\alpha} v_{L}^{(e)}\right] .
$$

Here, the integral of $f(p)$ in the module $|\cdots|^{2}$ is the spatial wave function at zero distance so that $|\cdots|^{2}=$ $|\Psi(0)|^{2} /\left(2 m_{e} m_{\mu}\right)$. The latter spinor product can be simplified into $2 m_{e} m_{\mu}$ in terms of their normalizations and spin combinations. Therefore, the mass mixing element arrives at

$$
m_{M \bar{M}}=16 \times \frac{G_{M \bar{M}}}{\sqrt{2}} \frac{|\Psi(0)|^{2}}{2}=16 \times \frac{G_{M \bar{M}}}{\sqrt{2}} \frac{(\mu \alpha)^{3}}{2 \pi},
$$

with the reduced mass $\mu=\frac{m_{e} m_{\mu}}{m_{e}+m_{\mu}} \approx m_{e}$. The mass eigenstates are then the simple combinations $\left|M_{1}\right\rangle=(|M\rangle+|\bar{M}\rangle) / \sqrt{2}$ and $\left|M_{2}\right\rangle=(|M\rangle-|\bar{M}\rangle) / \sqrt{2}$, and the difference in mass eigenvalues is given by $\Delta m \equiv m_{1}-m_{2}=2 m_{M \bar{M}}$. In experiments, muonium oscillation time is much longer than their decay time. So one can only hope to observe the mixing phenomenon by measuring the probability that a state that starts as a muonium $\left(\mu^{+} e^{-}\right)$decays as an antimuonium, where final states include a high energy electron and a low energy positron. Therefore, the state that starts as $|M\rangle$ at $t=0$ [denoted it by $|M(t)\rangle$ ] evolves as follows:

$$
|M(t)\rangle=\left|M_{1}\right\rangle\left\langle M_{1} \mid M\right\rangle e^{-i m_{1} t}+\left|M_{2}\right\rangle\left\langle M_{2} \mid M\right\rangle e^{-i m_{2} t},
$$

and will decay as a $\bar{M}$ with a total probability,

$$
\begin{aligned}
P(M \rightarrow \bar{M}) & =\int_{0}^{\infty} \frac{d t}{\tau} e^{-t / \tau}|\langle\bar{M} \mid M(t)\rangle|^{2}=\frac{(\Delta m \tau)^{2}}{2\left(1+(\Delta m \tau)^{2}\right)} \\
& \approx \frac{1}{2}(\Delta m \tau)^{2},
\end{aligned}
$$

where $\tau$ is the muon lifetime. Equation (7) does not take into account the effect of static electromagnetic fields in materials, which break the degeneracy $m_{M M}=m_{\bar{M} \bar{M}}$ and further suppresses the probability [22-24], an important effect in some experiments. In any case, we see from Eq. (7) that the conversion probability is in general very small and proportional to $\left|G_{M \bar{M}}\right|^{2}$,

$$
\begin{aligned}
P(M \rightarrow \bar{M}) & =\frac{64 G_{F}^{2} \alpha^{6} m_{e}^{6} \tau^{2}}{\pi^{2}}\left(\frac{G_{M \bar{M}}}{G_{F}}\right)^{2} \\
& =2.64 \times 10^{-5}\left(\frac{G_{M \bar{M}}}{G_{F}}\right)^{2} .
\end{aligned}
$$

The above formula is the transition probability without the magnetic field effect. In the magnetic field, the probability will be reduced by another factor of 0.35 based on Table II of Ref. [20]. It is easy to transform the PSI limit to that on the Wilson coefficient as $G_{M \bar{M}} / G_{F}<3 \times 10^{-3}$ at $90 \%$ C.L. The MACE experiment is expected to improve the sensitivity by at least 2 orders with $G_{M \bar{M}} / G_{F} \lesssim \mathcal{O}\left(10^{-5}\right)$, though the magnetic field correction factor is unknown yet and depends strongly on the design of detection systems.

\section{THE MUONIUM-ANTIMUONIUM CONVERSION IN SEESAW MODELS}

Now we explore the charged lepton flavor violation in the type-II seesaw model with the $M-\bar{M}$ transition. Especially, we would forecast the prospects of the MACE experiment to detect such a muonium-to-antimuonium conversion and constrain the parameter space in this popular neutrino mass model.

The type-II seesaw model, which is an extension of the standard model with a weak-scale triplet Higgs boson, is capable of generating small neutrino masses naturally. First of all, we briefly review the model in which the triplet Higgs boson possesses a weak scale mass, concentrating on how small neutrino masses are produced [25,26]. The triplet is arranged into an $S U(2)$ scalar multiplet denoted by $\Delta$ with a hypercharge $Y=1$,

$$
\Delta=\left(\begin{array}{cc}
\xi^{+} / \sqrt{2} & \xi^{++} \\
\xi^{0} & -\xi^{+} / \sqrt{2}
\end{array}\right) .
$$

The standard model gauge symmetry allows the Yukawa interaction between the lepton doublet $l=\left(\nu_{L}, e_{L}\right)^{T}$ and the triplet $\Delta$,

$$
\begin{aligned}
\mathcal{L}_{\mathrm{Yuk}}= & -\frac{1}{2}\left(y_{N}\right)_{i j} \bar{l}_{i}^{c} \varepsilon \Delta l_{j}+\text { H.c. } \\
= & -\frac{1}{2}\left(y_{N}\right)_{i j}\left[\bar{\nu}_{i}^{c} P_{L} \nu_{j} \xi^{0}-\frac{1}{\sqrt{2}}\left(\bar{\nu}_{i}^{c} P_{L} e_{j}+\bar{e}_{i}^{c} P_{L} \nu_{j}\right) \xi^{+}\right. \\
& \left.-\bar{e}_{i}^{c} P_{L} e_{j} \xi^{++}\right]+ \text {H.c. }
\end{aligned}
$$

where $\left(y_{N}\right)_{i j}$ are Yukawa coupling constants and the Latin indices $i, j$ represent generations and $\varepsilon \equiv i \sigma^{2}$.

It is easy to get $\left(y_{N}\right)_{i j}=\left(y_{N}\right)_{j i}$ and find that two terms of $\xi^{+}$are actually equal. The final Yukawa terms for $\Delta$ arrive at

$$
\begin{aligned}
\mathcal{L}_{\mathrm{Yuk}}= & \frac{1}{\sqrt{2}}\left[\xi^{+} \overline{\nu_{L}^{c}}\left(U_{\nu}^{T} y_{N}\right) e_{L}+\xi^{-} \overline{e_{L}}\left(y_{N}^{*} U_{\nu *}\right) \nu_{L}^{c}\right] \\
& +\frac{1}{2}\left[\xi^{++} \overline{e_{L}^{c}}\left(y_{N}\right) e_{L}+\xi^{--} \overline{e_{L}}\left(y_{N}^{*}\right) e_{L}^{c}\right] \\
& -\frac{1}{2}\left[\xi^{0 *} \overline{\nu_{L}^{c}}\left(y_{N}^{*}\right) \nu_{L}+\xi^{0} \overline{\nu_{L}}\left(y_{N}\right) \nu_{L}^{c}\right] \\
& -\frac{1}{2}\left[\overline{\nu_{L}^{c}}\left(m_{\nu}\right) \nu_{L}+\overline{\nu_{L}^{c}}\left(v_{3}^{*} U_{\nu} y_{N}^{*} U_{\nu}^{T}\right) \nu_{L}\right] .
\end{aligned}
$$

In addition to nonzero neutrino masses, the theory predicts the existence of three neutral, one charged, and one doubly 
charged physical Higgs scalar particles. Their masses and couplings with leptons and quarks depend crucially on the mechanism used to break the global U(1) symmetry associated with the conservation of lepton number L. In one realization is that the lepton number $\mathrm{L}$ is conserved by the Higgs potential $\mathrm{V}(\Phi, \Delta)$, while the U(1) global symmetry associated with the conservation of $\mathrm{L}$ is broken spontaneously as $\xi^{0}$ develops a nonzero vacuum expectation value (VEV). At this time, a linear combination of the imaginary parts of the neutral components of the Higgs doublet field $\Phi$ will act as a physical massless neutral scalar particle called a Majoron, which is almost ruled out in experiments. So we do not take this case into account. Interestingly, the other possibility is to assign two units of $\mathrm{L}$ to the Higgs triplet $\Delta\left(L_{\Delta}=-2\right)$ so that the Yukawa coupling terms $\left(\bar{l}^{c} \varepsilon \Delta l\right)$ would conserve the lepton number. The form of the U(1) symmetry breaking leading to $\left\langle\xi^{0}\right\rangle \neq 0$ and $\mathrm{L}$ nonconservation is determined by the assumed properties of the Higgs potential $\mathrm{V}(\Phi, \Delta)$ of the theory. The details of Higgs potential and the gauge sector can be found in the Appendix.

In addition to the triplet Higgs boson, we also consider the possibility that the neutrino masses get contributions from the other particles. In particular, we introduce a righthanded neutrino $\nu_{R}$ with additional couplings,

$$
\mathcal{L} \supset-y_{\nu i} \bar{L}_{i} \Phi \nu_{R}+M_{R} \nu_{R}^{c} \nu_{R}
$$

Then part of the neutrino mass matrix could be generated from the type-I seesaw mechanism. We take this model as the hybrid seesaw model.

While $\xi^{0}$ develops a vacuum expectation value $v_{3}$, the total Majorana neutrino masses are

$$
\left(m_{N}\right)_{i j}=\left(y_{N}\right)_{i j} v_{3}+y_{\nu i} y_{\nu j} \frac{v^{2}}{2 M_{R}} .
$$

The diagonalized neutrino masses is obtained by unitary transformations,

$\nu_{L} \rightarrow U_{\nu} \nu_{L}, \quad U_{\nu}^{T} m_{N} U_{\nu}=\operatorname{diag}\left\{m_{1}, m_{2}, m_{3}\right\} \equiv m_{\nu}$,

$m_{i} \geq 0$.

Without a loss of generality, we can work in a basis where charged lepton masses are diagonalized. Then $U_{\nu}$ is the Pontecorvo-Maki-Nakagawa-Sakata (PMNS) mixing matrix in charge-current interactions.

Given the Yukawa interaction in Eq. (10), it is easy to draw the leading-order contribution to the muoniumantimuonium conversion. As described in [9], if the usual Yukawa coupling of the lepton is assumed to be diagonal, we can obtain

$$
\begin{aligned}
\mathcal{H}_{\text {eff }} & =\frac{G_{M \bar{M}}}{\sqrt{2}}\left[\bar{\mu} \gamma^{\mu}\left(1-\gamma^{5}\right) e\right]\left[\bar{\mu} \gamma_{\mu}\left(1-\gamma^{5}\right) e\right], \quad \text { with } \\
G_{M \bar{M}} & =\frac{\left(y_{N}\right)_{e e}\left(y_{N}^{*}\right)_{\mu \mu}}{16 \sqrt{2} m_{++}^{2}} .
\end{aligned}
$$

The integrated probability that the muonium $M\left(\mu^{+} e^{-}\right)$ decay as $\mu^{-}$rather than $\mu^{+}$is

$$
\mathcal{P}(M \rightarrow \bar{M})=64^{3}\left(\frac{3 \pi^{2} \alpha^{3}}{G_{F} m_{\mu}^{2}}\right)^{2}\left(\frac{m_{e}}{m_{\mu}}\right)^{6}\left(\frac{G_{M \bar{M}}}{G_{F}}\right)^{2} .
$$

According to the latest experimental upper bound from PSI, we can obtain $\mathcal{P}(M \rightarrow \bar{M}) \leq 2.0 \times 10^{5} G_{M \bar{M}}^{2}$ at a $90 \%$ confidence level.

\section{CONSTRAINTS ON SEESAW MODELS}

The seesaw models considered in the present paper are strongly constrained by the lepton flavor violation (LFV) processes involving the charged lepton sector, such as $\ell_{a}^{ \pm} \rightarrow \ell_{b}^{\mp} \ell_{c}^{ \pm} \ell_{d}^{ \pm}$and $\ell_{a}^{ \pm} \rightarrow \ell_{b}^{ \pm} \gamma$. In both type-II seesaw and hybrid models, the leading-order contribution to $\ell_{a}^{ \pm} \rightarrow$ $\ell_{b}^{\mp} \ell_{c}^{ \pm} \ell_{d}^{ \pm}$is given by the tree-level diagram induced by the doubly charged scalar $\xi^{++}$. With the Yukawa couplings defined in Eq. (10), the corresponding branching ratios are given as follows [27]:

$\mathcal{B}\left(\ell_{a}^{ \pm} \rightarrow \ell_{b}^{\mp} \ell_{c}^{ \pm} \ell_{d}^{ \pm}\right)=\frac{1}{8\left(1+\delta_{c d}\right)} \frac{\left|\left(y_{N}\right)_{a b}\left(y_{N}^{\dagger}\right)_{c d}\right|^{2}}{G_{F}^{2} m_{++}^{4}}$,

where the Kronecker delta $\delta_{c d}$ accounts for identical leptons in the final states. In particular, the most precise channel in this class is provided by the decay $\mu^{+} \rightarrow$ $e^{+} e^{-} e^{+}$with the branching ratio given by [28-36]

$$
\mathcal{B}\left(\mu^{+} \rightarrow e^{+} e^{-} e^{+}\right)=\frac{\left|\left(y_{N}\right)_{\mu e}\left(y_{N}^{\dagger}\right)_{e e}\right|^{2}}{16 G_{F}^{2} m_{++}^{4}},
$$

which can be compared with the current best upper bound as follows [37]:

$$
\mathcal{B}\left(\mu^{+} \rightarrow e^{+} e^{-} e^{+}\right) \leq 1.0 \times 10^{-12} .
$$

For the type-II seesaw model, the LFV decay process $\mu \rightarrow e \gamma$ is generated at a one-loop level with the help of the doubly and singly charged scalars. As a result, the partial width for this process is given by $[27,29,31-36]$

$$
\mathcal{B}(\mu \rightarrow e \gamma) \simeq \frac{\alpha}{768 \pi} \frac{\left|\left(y_{N}^{\dagger} y_{N}\right)_{e \mu}\right|^{2}}{G_{F}^{2}}\left(\frac{1}{m_{+}^{2}}+\frac{8}{m_{++}^{2}}\right)^{2}
$$

where $\alpha$ refers to the electromagnetic fine structure constant, and in our derivation, we have ignored the dependence on the internal lepton masses since they are assumed 
to be much smaller than the $m_{+}$and $m_{++}$. On the other hand, for the hybrid model, the heavy singlet right-handed neutrino would mix with the $S U(2)_{L}$ active neutrinos and give rise to an additional one-loop contribution to $\mu \rightarrow e \gamma$ [34-36,38-48]. Even though this new LFV mode would be enhanced by the breaking of the Gloashow-IliopoulosMaina mechanism in the SM [38-48], it can be shown [34$36,47,48]$ that the corresponding amplitude is subdominant compared with those mediated by the charged scalars $\xi^{+}, \xi^{++}$and thus, can be safely ignored. Therefore, the dominant contribution to $\mu \rightarrow e \gamma$ remains to be the same as that of the type-II seesaw in Eq. (20). Nowadays, the most stringent constraint on $\mu \rightarrow e \gamma$ is given by the MEG Collaboration with the upper bound as follows [49]:

$$
\mathcal{B}(\mu \rightarrow \text { e } \gamma)<4.2 \times 10^{-13} .
$$

Later, we will use it to constrain the parameter spaces of both models in our numerical scanning.

The seesaw models considered in the present paper also suffer constraints from the $\mu \rightarrow e$ conversion in nuclei, which can arise from both short-range and long-range contributions. However, explicit calculations $[32,48]$ have shown that the current $\mu \rightarrow e$ conversion sensitivity cannot place any useful constraints on the parameter spaces, given already severe limits given by $\mu \rightarrow e \gamma$ and $\mu^{+} \rightarrow e^{+} e^{-} e^{+}$. Furthermore, the lepton number conservation in both models is broken by two units, and the obtained active neutrino masses are Majorana in nature. Thus, these models can be constrained by the lepton-number violating processes like neutrinoless double beta $(0 \nu \beta \beta)$ decays in nuclei, which so far have not yet been found. In the type-II seesaw model, there are two contributions to this process: one is the ordinary long-range channel by exchanging the light active neutrinos $\nu$, while the other is the short-range mode mediated by the doubly charged scalar $\xi^{ \pm \pm}$. However, these channels are severely suppressed either by the tiny light neutrino masses or by the doubly charged scalar mass scale $[35,50]$ and thus, can be neglected. The inclusion of one heavy singlet right-handed neutrino in the hybrid model does not alleviate the problem since the short-range mode induced by it suffers from a significant suppression arising from the extremely small heavy-light neutrino mixing. In summary, both models are insufficient to generate observable $0 \nu \beta \beta$ signals and thus, cannot be constrained by the limits from the existing experiments, like KamLAND-Zen [51] and GERDA [52].

The search for doubly charged Higgs boson has been presented at the LHC with the ATLAS detector [53], in which the analysis focused on the decays $\xi^{ \pm \pm} \rightarrow e^{ \pm} e^{ \pm}$, $\xi^{ \pm \pm} \rightarrow e^{ \pm} \mu^{ \pm}$, and $\xi^{ \pm \pm} \rightarrow \mu^{ \pm} \mu^{ \pm}$. The partial decay width of $\xi^{ \pm \pm}$to leptons is given by

$$
\Gamma\left(\xi^{ \pm \pm} \rightarrow \ell^{ \pm} \ell^{\prime \pm}\right)=k \frac{\left(y_{N}\right)_{\ell \ell^{\prime}}^{2}}{16 \pi} m_{++},
$$

where $k=2$ for $\ell=\ell^{\prime}$ and $k=1$ for $\ell \neq \ell^{\prime}$. Assuming $\xi^{ \pm \pm}$, almost all decay to leptons, i.e., $\sum_{\ell=e, \mu, \tau} \mathcal{B}\left(\xi^{ \pm \pm} \rightarrow\right.$ $\left.\ell^{ \pm} \ell^{\prime \pm}\right) \simeq 1$, we can get the branch ratios,

$$
\mathcal{B}\left(\xi^{ \pm \pm} \rightarrow \ell^{ \pm} \ell^{\prime \pm}\right)=\frac{k\left(y_{N}\right)_{\ell \ell^{\prime}}^{2}}{\sum_{\ell=e, \mu, \tau} k\left(y_{N}\right)_{\ell \ell^{\prime}}^{2}} .
$$

\section{NUMERICAL RESULTS}

In this section, we present our numerical results by sampling in the model parameter space. For simplicity, we assume all the model parameters are real. In our scanning, we selected models that predict the neutrino mass squared differences and mixing angles to be within the experimentally allowed ranges for both normal ordering (NO) and inverted ordering (IO) at the $3 \sigma$ confidence level obtained from the existing neutrino oscillation data as shown Table I. Although the recently released data from $\mathrm{T} 2 \mathrm{~K}$ and $\mathrm{NO} \nu \mathrm{A}$ Collaborations prefer a nonzero Dirac $C P$ phase $\delta_{\mathrm{CP}}$, this result is still not conclusive. Thus, we still allow $\delta_{\mathrm{CP}}$ to be varied within $[0,2 \pi]$. We also take into account the cosmological limit on the sum of the neutrino masses so that the lightest neutrino mass is sampled uniformly within the range of $[0,0.05 \mathrm{eV}]$. The value of the triplet Higgs $\mathrm{VEV}$ is adopted to be from $10^{-2} \mathrm{eV}$ to $1 \mathrm{GeV}$, while the mass doubly charged Higgs $\xi^{ \pm \pm}$is allowed to be within $[100 \mathrm{GeV}, 10 \mathrm{TeV}]$. Furthermore, we require that the selected models should satisfy the stringent bounds for cLFV processes, such as $\mu \rightarrow e \gamma$ and $\mu^{+} \rightarrow e^{-} e^{+} e^{+}$. For the remaining model parameters, we compare their predicted muonium-to-antimuonium conversion probability $\mathcal{P}(M \leftrightarrow \bar{M})$ with the existing PSI bound and the expected MACE sensitivity. In the following, we shall give the final scanning results for the type-II and hybrid seesaw models, respectively.

\section{A. Type II seesaw}

In this case, we assume all of the neutrino mass comes from the triplet Higgs boson, while the contribution from the right-handed neutrino can be negligible. In Fig. 1, we

TABLE I. The allowed ranges of the neutrino mass square differences and mixing angles at $3 \sigma$ confidence level for normal ordering and inverted ordering used in our numerical analysis [54]. Note that $\Delta m_{3 \ell}^{2} \equiv \Delta m_{31}^{2}>0$ for normal ordering and $\Delta m_{3 \ell}^{2} \equiv \Delta m_{32}^{2}<0$ for inverted ordering.

\begin{tabular}{lcc}
\hline \hline & Normal ordering & Inverted ordering \\
\hline $\sin ^{2} \theta_{12}$ & $0.269-0.343$ & $0.269-0.343$ \\
$\sin ^{2} \theta_{23}$ & $0.407-0.618$ & $0.411-0.621$ \\
$\sin ^{2} \theta_{13}$ & $0.02034-0.02430$ & $0.02053-0.02436$ \\
$\frac{\Delta m_{21}^{2}}{10^{-5} \mathrm{eV}^{2}}$ & $6.82-8.04$ & $6.82-8.04$ \\
$\frac{\Delta m_{3 \ell}^{2}}{10^{-3} \mathrm{eV}^{2}}$ & $+2.431-+2.598$ & $-2.583--2.412$ \\
\hline \hline
\end{tabular}



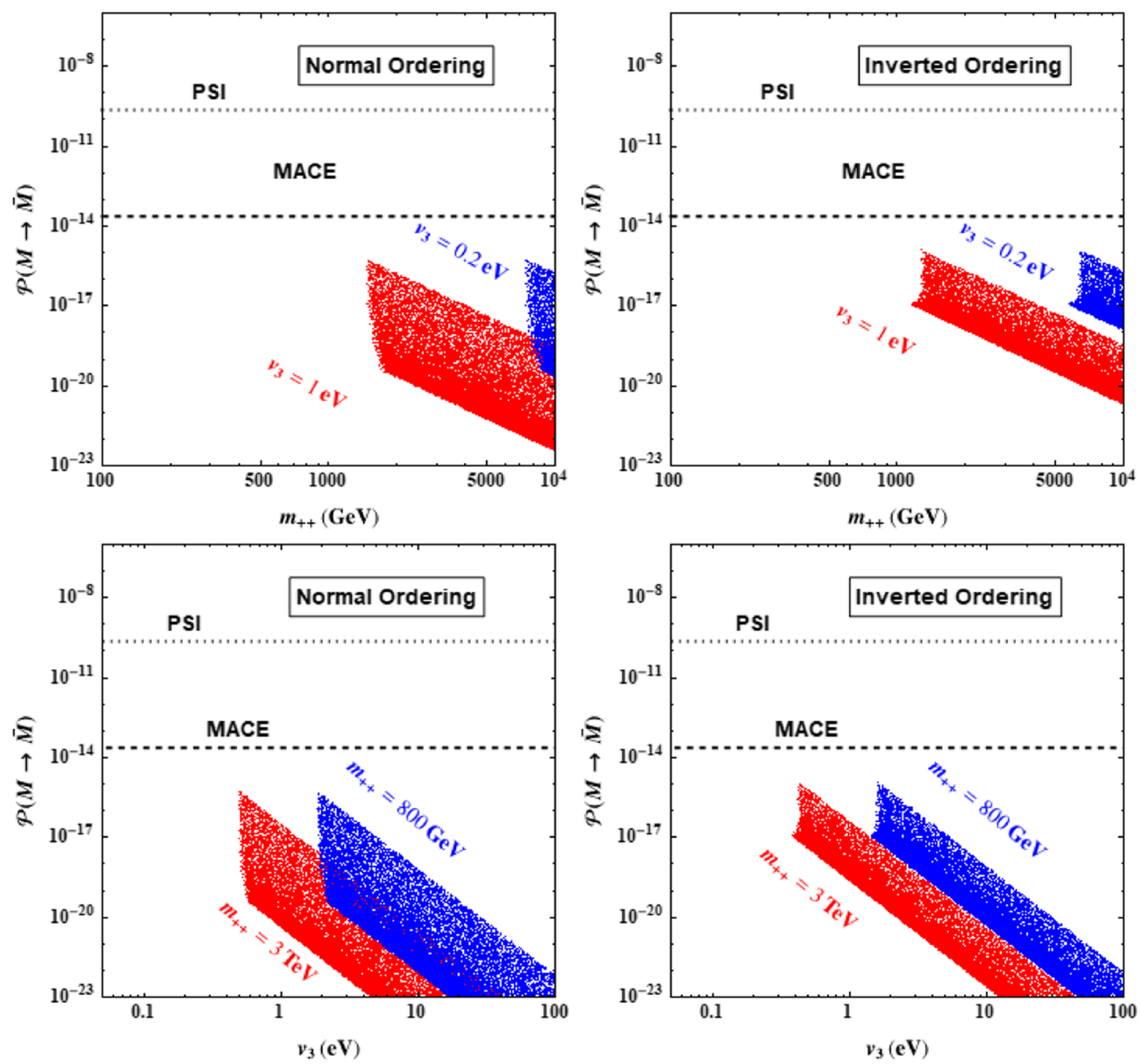

FIG. 1. Upper: Scatter plots of the doubly charged Higgs mass $m_{++}$and the conversion probability $P$ without magnetic field correction in the type-II seesaw with $v_{3}=0.2 \mathrm{eV}$ (blue points) and $v_{3}=1 \mathrm{eV}$ (red points) for normal ordering (left) and inverted ordering (right). Lower: Scatter plots of the VEV $v_{3}$ and the conversion probability $P$ in the type-II seesaw with $m_{++}=800 \mathrm{GeV}$ (blue points) and $m_{++}=3 \mathrm{TeV}$ (red points) for normal ordering (left) and inverted ordering (right). The PSI bound is transformed into the one at zero magnetic field at $90 \%$ C.L. All the plotted points are allowed by the flavor and collider constraints.

show the scan result by requiring the neutrino mass satisfying the observation. Since there is an ambiguity in the neutrino mass ordering, we split our analysis in these two cases. The left panels show the preferred parameter space for neutrino mass normal ordering and the right panels that of inverted ordering. For the top two plots, we fixed the vacuum value of the triplet Higgs boson at $1 \mathrm{eV}$ (red) and $0.2 \mathrm{eV}$ (blue). We find for $v_{3}=1 \mathrm{eV}$, the doubly charged Higgs boson should be heavier than around $1.5 \mathrm{TeV}$ to evade the limit from flavor constraints, particularly from $\mu^{+} \rightarrow e^{-} e^{+} e^{+}$. In the case of $v_{3}=0.2 \mathrm{eV}$, the limit on the doubly charged Higgs boson is stronger since a larger Yukawa coupling is needed to generate the neutrino mass for a smaller vacuum value of $\Delta$. We note that the preferred parameter space behaves as a band due to the uncertainty of the neutrino mass parameter. Finally, we find all the survived parameter space predicts a muonium-antimuonium conversion probability less than $10^{-15}$, which is beyond the reach of the future MACE experiment.

\section{B. Hybrid seesaw}

As shown in previous model, the strongest limit arises from the cLFV processes, such as $\mu^{+} \rightarrow e^{+} \gamma$ and $\mu^{+} \rightarrow e^{-} e^{+} e^{+}$. The reason is that from the PMNS matrix we can see the neutrino mixing is relatively large, and large off diagonal terms in the neutrino mass matrix are needed. Since the neutrino mass matrix mainly originates from the triplet Higgs boson, it requires a large flavor changing coupling between the triplet Higgs boson and the leptons, inducing a large flavor changing effect. In the hybrid seesaw model, the flavor constrains could be weaker due to the contribution of off diagonal terms from a right-handed neutrino. Specifically, here we consider that all off diagonal 

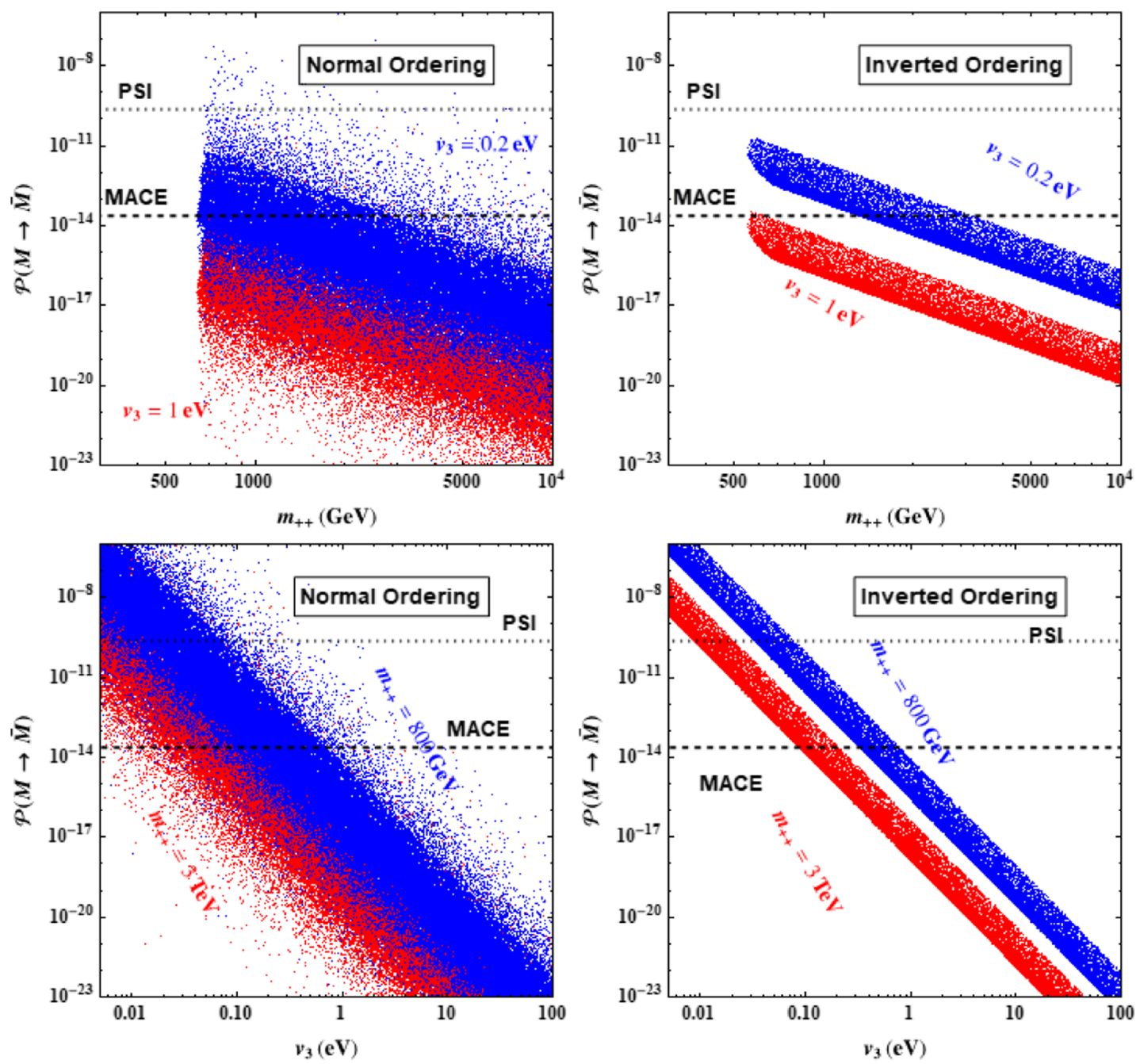

FIG. 2. Upper: Scatter plots of the doubly charged Higgs mass $m_{++}$and the conversion probability $P$ without magnetic field correction in the hybrid seesaw model with $v_{3}=0.2 \mathrm{eV}$ (blue points) and $v_{3}=1 \mathrm{eV}$ (red points) for normal ordering (left) and inverted ordering (right). Lower: Scatter plots of the VEV $v_{3}$ and the conversion probability $P$ in the hybrid seesaw model with $m_{++}=800 \mathrm{GeV}$ (blue points) and $m_{++}=3 \mathrm{TeV}$ (red points) for normal ordering (left) and inverted ordering (right). The PSI bound is transformed into the one at zero magnetic field at $90 \%$ C.L. All the plotted points are allowed by the flavor and collider constraints.

terms of the neutrino mass matrix originate from the righthanded neutrino; thus, all of Yukawa couplings $y_{\nu}$ are fixed. Hence, all the flavor constraints could be removed ${ }^{1}$.

The final scanning results are shown in Fig. 2. As before, the upper two scatter plots show the muonium-to-antimuonium conversion probability $\mathcal{P}(M \leftrightarrow \bar{M})$ as a function of the doubly charged Higgs mass $m_{++}$for both NO (left panel) and IO (right panel), while the lower two ones correspond to parameter spaces in $\mathcal{P}(M \leftrightarrow \bar{M})$ vs $v_{3}$ plane. Compared with the previous type-II seesaw case, it is clear that the allowed parameter spaces for the hybrid seesaw model are enlarged greatly, extending aggressively into the low doubly charged Higgs mass region and the low triplet

\footnotetext{
${ }^{1}$ The contribution of the flavor changing observable from the right-handed neutrino can be safely removed assuming the righthanded neutrino is heavy enough.
}

VEV $v_{3}$ region. Due to the relax of cLFV constraints in this hybrid model, the lower bounds on the doubly charged Higgs mass for both $\mathrm{NO}$ and IO cases around $600 \mathrm{GeV}$ are given by the collider searches for $\xi^{ \pm \pm}$. As a result, it is seen that MACE can increase the detection region considerably. Concretely, for $v_{3}=0.2 \mathrm{eV}$, MACE can detect a doubly charged Higgs boson as heavy as $3 \mathrm{TeV}$. On the other hand, for a fixed $m_{++}$, MACE has an ability to measure the parameter region with $v_{3}<1 \mathrm{eV}$ for both orderings, which is almost 1 order more sensitive than the PSI detector. The study in this subsection demonstrates the advantage and the necessity of MACE in the probing and constraining the triplet scalar in the seesaw models.

\section{SUMMARY AND CONCLUSION}

In the present paper, we have discussed prospects of the proposed MACE experiment to search for the 
muonium-to-antimuonium conversion process in the typeII and hybrid seesaw models, the latter of which is an extension of the type-II seesaw by including a single heavy right-handed neutrino. Note that the leading-order contributions to the $M-\bar{M}$ conversion probability in both models are induced at tree level by the doubly charged scalar originated in the $S U(2)_{L}$ triplet scalar. Thus, MACE can effectively measure the parameter space related to this triplet scalar. By utilizing the high-quality slow muon sources at CSNS, together with other significant advances in the detector technologies, MACE has been shown to be able to improve the sensitivity to the conversion probability by almost 3 orders of magnitude compared with the existing PSI experiment accomplished more than 20 years ago. Therefore, a large uncharted model parameter space is expected to be probed by MACE, which is complementary to direct collider searches, neutrino oscillations, and other cLFV channels such as $\mu^{+} \rightarrow e^{+} \gamma$ and $\mu^{+} \rightarrow e^{-} e^{+} e^{+}$. As a result, it is shown that MACE is not sensitive to the parameter space of interest surviving from the collider and flavor limits. On the other hand, for the hybrid seesaw model, MACE can reach unexplored parameter region and thus provides useful information on the triplet scalar complementary to other cLFV experiments like MEG-II and Mu3e. In other words, if no positive signals are observed by MACE in the future, we shall be able to set a much more stringent upper bound on the mass of the doubly charged scalar around $1.2 \mathrm{TeV}$ for $v_{3}=1 \mathrm{eV}$ in the case of inverted ordering, which will surpass the corresponding LHC limits even by taking into account the uncertainties involving the neutrino mass ordering or the VEV of the triplet scalar. Even for the NO, the prospect of MACE can reach a doubly charged Higgs boson as heavy as $3 \mathrm{TeV}$ for $v_{3}=1 \mathrm{eV}$. In summary, with the advent of MACE, the muonium-to-antimuonium conversion will become one of golden channels to study the cLFV physics, which, together with other flavor and collider searches, will shed light on the mystery of the neutrino masses.

\section{ACKNOWLEDGMENTS}

This work was supported in part by National Natural Science Foundation of China under Grant No. 12075326, and Guangdong Basic and Applied Basic Research Foundation under Grant No. 2019A1515012216, Original innovation project of Chinese Academy of Sciences (CAS) under Grant No. ZDBS-LY-SLH009 and CAS Center for Excellence in Particle Physics (CCEPP). JT appreciates fruitful discussions with the accelerator group of EMuS.C.H. is supported by SYSU startup funding. D. H. is supported by National Science Foundation of China (NSFC) under Grant No. 12005254. Y.Z. is supported by National Science Foundation of China (NSFC) under Grants No. 11805001 and No. 11935001. We finally acknowledge Dr. Sampsa Vihonen's help in English proofreading.

\section{APPENDIX A: HIGGS POTENTIAL}

We consider the general Higgs potential for a doublet $\Phi=\left(h^{+}, h^{0}\right)^{T}$ and a triplet Higgs $\Delta[55]$,

$$
\begin{aligned}
V(\Phi, \Delta)= & m^{2}\left(\Phi^{\dagger} \Phi\right)+\lambda_{1}\left(\Phi^{\dagger} \Phi\right)^{2}+M_{\Delta^{2}} \operatorname{Tr}\left(\Delta^{\dagger} \Delta\right) \\
& +\lambda_{2}\left[\operatorname{Tr}\left(\Delta^{\dagger} \Delta\right)\right]^{2}+\lambda_{3} \operatorname{Tr}(\Delta \Delta) \operatorname{Tr}\left(\Delta^{\dagger} \Delta^{\dagger}\right), \\
& +\lambda_{4}\left(\Phi^{\dagger} \Phi\right)\left[\operatorname{Tr}\left(\Delta^{\dagger} \Delta\right)\right]+\lambda_{5}\left(\Phi^{\dagger} \Delta^{\dagger} \Delta \Phi\right) \\
& +\lambda_{6}\left(\Phi^{\dagger} \Delta \Delta^{\dagger} \Phi\right)+\frac{1}{2} \mu\left(\Phi^{T} \varepsilon \Delta^{\dagger} \Phi\right)+\text { H.c. }
\end{aligned}
$$

The breaking can be explicit when $\mathrm{V}(\Phi, \Delta)$ is supposed to contain the trilinear L-nonconserving term $\left(\Phi^{T} \Delta \Phi\right)$. In this case, the masses of the new Higgs particles $M_{\Delta}$ in the model can be arbitrary large. Collect the following soft lepton number violating trilinear interaction between $\Delta$ and $\Phi$ from the Higgs potential:

$$
\begin{aligned}
\mathcal{L}_{\text {soft }}= & -\frac{1}{2} \mu \Phi^{T} \varepsilon \Delta^{\dagger} \Phi+\text { H.c., } \\
= & -\frac{1}{2} \mu\left[\left(h^{+}\right)^{2} \xi^{--}-\sqrt{2} h^{+} h^{0} \xi^{-}-\left(h^{0}\right)^{2} \xi^{0 \dagger}\right] \\
& + \text { H.c., }
\end{aligned}
$$

where $\mu$ is an undetermined parameter. The minimum of Higgs potential is presented in the following:

$$
\begin{aligned}
V_{\min }(\Phi, \Delta)= & \frac{1}{2} m^{2} v_{1}^{2}+M_{\Delta}^{2} v_{3}^{2}+\frac{1}{4} \lambda_{1} v_{1}^{4}+\lambda_{2} v_{3}^{4}+\frac{1}{2} \lambda_{4} v_{1}^{2} v_{3}^{2} \\
& +\frac{1}{2} \lambda_{6} v_{1}^{2} v_{3}^{2}+\frac{1}{2} \mu v_{1}^{2} v_{3} .
\end{aligned}
$$

After the spontaneous symmetry breaking (SSB), solving the equation $\frac{\partial V_{\min }(\Phi, \Delta)}{\partial v_{i}}=0$ leads to

$$
\begin{aligned}
\left\langle h^{0}\right\rangle & =\frac{v_{1}}{\sqrt{2}}, \quad\left\langle\xi^{0}\right\rangle=v_{3}, \\
v_{1}^{2} & =-\frac{m^{2}}{\lambda_{1}}, \quad v_{3}=-\frac{\mu v_{1}^{2}}{2\left(2 M^{2}+v_{1}^{2} \lambda_{4}+v_{1}^{2} \lambda_{6}\right)},
\end{aligned}
$$

where $\mu<0, m^{2}<0$ and $M_{\Delta}^{2}>0$. When $|\mu|$ is small enough compared with the weak scale, the smallness of neutrino masses is attributed to the tiny $\mu$, which is estimated at the $\mathrm{eV}$ scale in the case, where $y_{N} \sim O(1)$ and $M_{\Delta} \sim v_{1}$. For completeness, the charged Higgs masses are listed below,

$$
\begin{gathered}
m_{+}=M_{\Delta}^{2}+\frac{1}{2}\left(\lambda_{4}+\frac{\lambda_{5}+\lambda_{6}}{2}\right) v_{1}^{2} \\
m_{++}=M_{\Delta}^{2}+\frac{1}{2}\left(\lambda_{4}+\lambda_{5}\right) v_{1}^{2} .
\end{gathered}
$$




\section{APPENDIX B: GAUGE SECTOR}

Now let us move to gauge couplings $D_{\mu} \xi D^{\mu} \xi$. Since there is a triplet scalar, we have to use a three-dimension representation of the $\mathrm{SU}(2)$ group,

$$
\begin{aligned}
T_{1} & =\left(\begin{array}{lll}
0 & \frac{1}{\sqrt{2}} & 0 \\
\frac{1}{\sqrt{2}} & 0 & \frac{1}{\sqrt{2}} \\
0 & \frac{1}{\sqrt{2}} & 0
\end{array}\right), \quad T_{2}=\left(\begin{array}{ccc}
0 & -\frac{i}{\sqrt{2}} & 0 \\
\frac{i}{\sqrt{2}} & 0 & -\frac{i}{\sqrt{2}} \\
0 & \frac{i}{\sqrt{2}} & 0
\end{array}\right), \\
T_{3} & =\left(\begin{array}{ccc}
1 & 0 & 0 \\
0 & 0 & 0 \\
0 & 0 & -1
\end{array}\right) \\
T^{+} & =T^{1}+i T^{2}=\left(\begin{array}{ccc}
0 & \sqrt{2} & 0 \\
0 & 0 & \sqrt{2} \\
0 & 0 & 0
\end{array}\right), \\
T^{-} & =T^{1}-i T^{2}=\left(\begin{array}{ccc}
0 & 0 & 0 \\
\sqrt{2} & 0 & 0 \\
0 & \sqrt{2} & 0
\end{array}\right)
\end{aligned}
$$

$$
\begin{gathered}
D_{\mu} \xi=\partial_{\mu}-i g_{1} Y_{\xi} B_{\mu}-i g_{2} A_{\mu}^{a} T^{a} \\
=\left(\begin{array}{c}
\left(\partial_{\mu}-i B_{\mu} g_{1}-i A_{\mu}^{3} g_{2}\right) \xi^{++}-i g_{2} W_{\mu}^{+} \xi^{+} \\
\left(\partial_{\mu}-i B_{\mu} g_{1}\right) \xi^{+}-i g_{2} W_{\mu}^{-} \xi^{++}-i g_{2} W_{\mu}^{+} \xi^{0} \\
\left(\partial_{\mu}-i B_{\mu} g_{1}+i A_{\mu}^{3} g_{2}\right) \xi^{0}-i g_{2} W_{\mu}^{-} \xi^{+}
\end{array}\right) \\
W_{\mu}^{ \pm}=\frac{1}{\sqrt{2}}\left(A_{\mu}^{1} \mp i A_{\mu}^{2}\right) \\
s_{w} \equiv \sin \theta_{w}=\frac{g_{1}}{\sqrt{g_{1}^{2}+g_{2}^{2}}} \\
c_{w} \equiv \cos \theta_{w}=\frac{g_{2}}{\sqrt{g_{1}^{2}+g_{2}^{2}}} \\
A_{\mu}^{3}=Z_{\mu}^{0} \cos \theta_{w}+A_{\mu} \sin \theta_{w} \\
B_{\mu}=-Z_{\mu}^{0} \sin \theta_{w}+A_{\mu} \cos \theta_{w} .
\end{gathered}
$$

After spontaneous symmetry breaking, $\Delta$ develops a vacuum expectation value. If physical vector bosons are introduced, we have

$$
D^{\mu *}(\xi+\langle\xi\rangle)=\left(\begin{array}{l}
i W^{-\mu} \xi^{-} g_{2}+\left[i g_{2}\left(Z^{0 \mu} c_{w}+A^{\mu} s_{w}\right)+i g_{1}\left(A^{\mu} c_{w}-Z^{0 \mu} s_{w}\right)+\partial^{\mu}\right] \xi^{--} \\
i A^{\mu} \xi^{-} c_{w} g_{1}+i g_{2}\left(W^{+\mu} \xi^{--}+W^{-\mu} \xi^{0 *}\right)+\left(\partial^{\mu}-i Z^{0 \mu} g_{1} s_{w}\right) \xi^{-} \\
i W^{+\mu} \xi^{-} g_{2}+\left[-i g_{2}\left(Z^{\mu \mu} c_{w}+A^{\mu} s_{w}\right)+i g_{1}\left(A^{\mu} c_{w}-Z^{0 \mu} s_{w}\right)+\partial^{\mu}\right] \xi^{0 *}
\end{array}\right)
$$

They will contribute to gauge boson masses,

$$
\begin{aligned}
\left|D_{\mu}\langle\xi\rangle\right|^{2}= & W_{\mu}^{+} W^{-\mu} g_{2}^{2} v_{3}^{2}+Z_{\mu}^{0} Z^{0 \mu}\left(c_{w}^{2} g_{2}^{2}+2 c_{w} g_{1} s_{w} g_{2}+g_{1}^{2} s_{w}^{2}\right) v_{3}^{2} \\
& +Z_{\mu}^{0} A^{\mu}\left(-g_{1} g_{2} c_{w}^{2}-g_{1}^{2} s_{w} c_{w}+g_{2}^{2} s_{w} c_{w}+g_{1} g_{2} s_{w}^{2}-g_{1} g_{2} c_{w}^{2}-g_{1}^{2} s_{w} c_{w}+g_{2}^{2} s_{w} c_{w}+g_{1} g_{2} s_{w}^{2}\right) v_{3}^{2} \\
& +A_{\mu} A^{\mu}\left(c_{w}^{2} g_{1}^{2}-2 c_{w} g_{2} s_{w} g_{1}+g_{2}^{2} s_{w}^{2}\right) v_{3}^{2} \\
= & g_{2}^{2} v_{3}^{2} W^{+\mu} W_{\mu}^{-}+\frac{g_{2}^{2} v_{3}^{2}}{\cos ^{2} \theta_{w}} Z^{0 \mu} Z_{\mu}^{0} .
\end{aligned}
$$

In addition, the traditional SM contains gauge mass terms,

$$
\mathcal{L}_{\mathrm{GM}}=\frac{1}{4} g_{2}^{2} v_{1}^{2} W_{\mu}^{+} W^{-\mu}+\frac{1}{8} \frac{g_{2}^{2} v_{1}^{2}}{\cos ^{2} \theta_{w}} Z_{\mu}^{0} Z^{0 \mu},
$$

which violates the custodial symmetry such as

$$
\rho=\frac{M_{W}^{2}}{M_{Z}^{2} \cos ^{2} \theta_{w}}=\frac{\frac{1}{4} g_{2}^{2} v_{1}^{2}+g_{2}^{2} v_{3}^{2}}{\frac{1}{4} g_{2}^{2} v_{1}^{2}+2 g_{2}^{2} v_{3}}=1-\frac{v_{3}^{2}}{\frac{1}{4} v_{1}^{2}+2 v_{3}^{2}} .
$$

As $|\mu|$ is small enough so that $v_{3} \ll v_{1}, \rho \approx 1$ meets the requirement from electroweak precision experiments whereas detectable lepton flavor violating processes are expected. Since the lepton number is restored for $\mu=0$, it may be natural to have a small $\mu$ as a consequence of a tiny lepton number violation. In fact, there is a tree-level lepton flavor violating process $e^{-} \mu^{+} \rightarrow e^{+} \mu^{-}$mediated by $\xi^{++}$ due to the interaction term $\bar{e}_{i}^{c} P_{L} e_{j} \xi^{++}$[9] just as the related term of Eq. (10). 
[1] N. Berger, The Mu3e experiment, Nucl. Phys. B, Proc. Suppl. 248-250, 35 (2014).

[2] A. M. Baldini et al., The design of the MEG II experiment, Eur. Phys. J. C 78, 380 (2018).

[3] R. Abramishvili et al., COMET Phase-I technical design report, Prog. Theor. Exp. Phys. 2020, 033C01 (2020).

[4] L. Bartoszek et al., Mu2e technical design report, arXiv: 1501.05241.

[5] B. Pontecorvo, Mesonium and anti-mesonium, Sov. Phys. JETP 6, 429 (1957), https://inspirehep.net/literature/2884.

[6] T. E. Clark and S. T. Love, Muonium - anti-muonium oscillations and massive Majorana neutrinos, Mod. Phys. Lett. A 19, 297 (2004).

[7] G. Cvetic, C. O. Dib, C. S. Kim, and J. D. Kim, Muoniumantimuonium conversion in models with heavy neutrinos, Phys. Rev. D 71, 113013 (2005).

[8] B. Liu, Gauge invariance of the muonium-antimuonium oscillation time scale and limits on right-handed neutrino masses, Mod. Phys. Lett. A 24, 335 (2009).

[9] D. Chang and W.-Y. Keung, Constraints on Muonium Anti-Muonium Conversion, Phys. Rev. Lett. 62, 2583 (1989).

[10] H. Fujii, Y. Mimura, K. Sasaki, and T. Sasaki, Muonium, hyperfine structure and the decay mu+- $>$ e+ + antielectron-neutrino + muon-neutrino in models with dilepton gauge baryons, Phys. Rev. D 49, 559 (1994).

[11] P. H. Frampton and M. Harada, Constraints from precision electroweak data on leptoquarks and bileptons, Phys. Rev. D 58, 095013 (1998).

[12] A. Halprin and A. Masiero, Muonium - anti-muonium oscillations and exotic muon decay in broken R-parity SUSY models, Phys. Rev. D 48, R2987 (1993).

[13] P. Herczeg and R. N. Mohapatra, Muonium to Anti-Muonium Conversion and the Decay $\mathrm{mu}+\longrightarrow \mathrm{e}+$ Anti-Neutrino Neutrino in Left-Right Symmetric Models, Phys. Rev. Lett. 69, 2475 (1992).

[14] A. Halprin, Neutrinoless Double Beta Decay and Muonium-Anti-Muonium Transitions, Phys. Rev. Lett. 48, 1313 (1982).

[15] A. Crivellin, M. Ghezzi, L. Panizzi, G. M. Pruna, and A. Signer, Low- and high-energy phenomenology of a doubly charged scalar, Phys. Rev. D 99, 035004 (2019).

[16] T. Li and M.A. Schmidt, Sensitivity of future lepton colliders and low-energy experiments to charged lepton flavor violation from bileptons, Phys. Rev. D 100, 115007 (2019).

[17] Y. Du, A. Dunbrack, M. J. Ramsey-Musolf, and J.-H. Yu, Type-II Seesaw scalar triplet model at a $100 \mathrm{TeV} p p$ collider: Discovery and Higgs portal coupling determination, J. High Energy Phys. 01 (2019) 101.

[18] R. Padhan, D. Das, M. Mitra, and A. K. Nayak, Probing doubly and singly charged Higgs bosons at the $p p$ collider HE-LHC, Phys. Rev. D 101, 075050 (2020).

[19] R. Conlin and A. A. Petrov, Muonium-antimuonium oscillations in effective field theory, Phys. Rev. D 102, 095001 (2020).

[20] L. Willmann et al., New Bounds from Searching for Muonium to Anti-Muonium Conversion, Phys. Rev. Lett. 82, 49 (1999).
[21] J. Tang et al., Letter of interest contribution to snowmass21, https://www.snowmass21.org/docs/files/summaries/RF/ SNOWMASS21-RF5_RF0_Jian_Tang-126.pdf.

[22] G. Feinberg and S. Weinberg, Conversion of muonium into antimuonium, Phys. Rev. 123, 1439 (1961).

[23] W.-S. Hou and G.-G. Wong, Magnetic field dependence of muonium - anti-muonium conversion, Phys. Lett. B 357, 145 (1995).

[24] K. Horikawa and K. Sasaki, Muonium - anti-muonium conversion in models with dilepton gauge bosons, Phys. Rev. D 53, 560 (1996).

[25] J. Schechter and J. W. F. Valle, Neutrino masses in $\mathrm{SU}(2) \times \mathrm{U}(1)$ theories, Phys. Rev. D 22, 2227 (1980).

[26] M. Kakizaki and M. Yamaguchi, Singular Kahler potential and heavy top quark in a democratic mass matrix model, Phys. Lett. B 573, 123 (2003).

[27] R. Primulando, J. Julio, and P. Uttayarat, Scalar phenomenology in type-II seesaw model, J. High Energy Phys. 08 (2019) 024.

[28] P. B. Pal, Constraints on a muon - neutrino mass around 100-kev, Nucl. Phys. B227, 237 (1983).

[29] G. K. Leontaris, K. Tamvakis, and J. D. Vergados, Lepton and family number violation from exotic scalars, Phys. Lett. 162B, 153 (1985).

[30] M. L. Swartz, Limits on doubly charged Higgs bosons and lepton flavor violation, Phys. Rev. D 40, 1521 (1989).

[31] R. N. Mohapatra, Rare decays of the tau lepton as a probe of the left-right symmetric theories of weak interactions, Phys. Rev. D 46, 2990 (1992).

[32] V. Cirigliano, A. Kurylov, M. J. Ramsey-Musolf, and P. Vogel, Lepton flavor violation without supersymmetry, Phys. Rev. D 70, 075007 (2004).

[33] A. G. Akeroyd, M. Aoki, and H. Sugiyama, Lepton flavour violating decays tau $\longrightarrow>$ anti-l 11 and $\mathrm{mu} \longrightarrow>$ e gamma in the Higgs triplet model, Phys. Rev. D 79, 113010 (2009).

[34] D. N. Dinh, A. Ibarra, E. Molinaro, and S. T. Petcov, The $\mu-e$ conversion in nuclei, $\mu \rightarrow e \gamma, \mu \rightarrow 3 e$ decays and $\mathrm{TeV}$ scale see-saw scenarios of neutrino mass generation, J. High Energy Phys. 08 (2012) 125; Erratum, J. High Energy Phys. 09 (2013) 23.

[35] P. S. Bhupal Dev, M. J. Ramsey-Musolf, and Y. Zhang, Doubly-charged scalars in the Type-II seesaw mechanism: Fundamental symmetry tests and high-energy searches, Phys. Rev. D 98, 055013 (2018).

[36] M. M. Ferreira, T. B. de Melo, S. Kovalenko, P. R. D. Pinheiro, and F.S. Queiroz, Lepton flavor violation and collider searches in a Type I + II seesaw model, Eur. Phys. J. C 79, 955 (2019).

[37] U. Bellgardt et al., Search for the decay mu+- $>$ e+ e+ e-, Nucl. Phys. B299, 1 (1988).

[38] P. Minkowski, $\mu \rightarrow e \gamma$ at a rate of one out of $10^{9}$ muon decays?, Phys. Lett. 67B, 421 (1977).

[39] C. S. Lim and T. Inami, Lepton flavor nonconservation and the mass generation mechanism for neutrinos, Prog. Theor. Phys. 67, 1569 (1982).

[40] P. Langacker and D. London, Lepton number violation and massless nonorthogonal neutrinos, Phys. Rev. D 38, 907 (1988).

[41] W. J. Marciano and A. I. Sanda, Exotic decays of the muon and heavy leptons in gauge theories, Phys. Lett. 67B, 303 (1977). 
[42] T. P. Cheng and L.-F. Li, $\mu \rightarrow e \gamma$ in Theories With Dirac and Majorana Neutrino Mass Terms, Phys. Rev. Lett. 45, 1908 (1980).

[43] A. Ilakovac and A. Pilaftsis, Flavor violating charged lepton decays in seesaw-type models, Nucl. Phys. B437, 491 (1995).

[44] F. Deppisch and J. W. F. Valle, Enhanced lepton flavor violation in the supersymmetric inverse seesaw model, Phys. Rev. D 72, 036001 (2005).

[45] B. He, T. P. Cheng, and L.-F. Li, A less suppressed mu-> e gamma loop amplitude and extra dimension theories, Phys. Lett. B 553, 277 (2003).

[46] L. Lavoura, General formulae for $\mathrm{f}(1) \longrightarrow \mathrm{f}(2)$ gamma, Eur. Phys. J. C 29, 191 (2003).

[47] D. V. Forero, S. Morisi, M. Tortola, and J. W. F. Valle, Lepton flavor violation and non-unitary lepton mixing in low-scale type-I seesaw, J. High Energy Phys. 09 (2011) 142.

[48] R. Alonso, M. Dhen, M. B. Gavela, and T. Hambye, Muon conversion to electron in nuclei in type-I seesaw models, J. High Energy Phys. 01 (2013) 118.

[49] A. M. Baldini et al., Search for the lepton flavour violating decay $\mu^{+} \rightarrow \mathrm{e}^{+} \gamma$ with the full dataset of the MEG experiment, Eur. Phys. J. C 76, 434 (2016).
[50] J. Chakrabortty, H. Zeen Devi, S. Goswami, and S. Patra, Neutrinoless double- $\beta$ decay in TeV scale Left-Right symmetric models, J. High Energy Phys. 08 (2012) 008.

[51] A. Gando et al., Search for Majorana Neutrinos near the Inverted Mass Hierarchy Region with KamLAND-Zen, Phys. Rev. Lett. 117, 082503 (2016); Erratum, Phys. Rev. Lett. 117, 109903 (2016).

[52] M. Agostini et al., Improved Limit on Neutrinoless Double$\beta$ Decay of ${ }^{76} \mathrm{Ge}$ from GERDA Phase II, Phys. Rev. Lett. 120, 132503 (2018).

[53] M. Aaboud et al., Search for doubly charged Higgs boson production in multi-lepton final states with the ATLAS detector using proton-proton collisions at $\sqrt{s}=13 \mathrm{TeV}$, Eur. Phys. J. C 78, 199 (2018).

[54] I. Esteban, M. C. Gonzalez-Garcia, M. Maltoni, T. Schwetz, and A. Zhou, The fate of hints: Updated global analysis of three-flavor neutrino oscillations, J. High Energy Phys. 09 (2020) 178.

[55] E. J. Chun, K. Y. Lee, and S. C. Park, Testing Higgs triplet model and neutrino mass patterns, Phys. Lett. B 566, 142 (2003). 